\title{
Spinal and Pontine Relay Pathways Mediating Respiratory Rhythm Entrainment by Limb Proprioceptive Inputs in the Neonatal Rat
}

\author{
Aurore Giraudin, Morgane Le Bon-Jégo, Marie-Jeanne Cabirol, John Simmers, ${ }^{*}$ and Didier Morin* \\ Université Bordeaux, Institut de Neurosciences Cognitives et Intégratives d'Aquitaine, Centre National de la Recherche Scientifique Unité Mixte de \\ Recherche 5287, F-33000 Bordeaux, France
}

\begin{abstract}
The coordination of locomotion and respiration is widespread among mammals, although the underlying neural mechanisms are still only partially understood. It was previously found in neonatal rat that cyclic electrical stimulation of spinal cervical and lumbar dorsal roots (DRs) can fully entrain (1:1 coupling) spontaneous respiratory activity expressed by the isolated brainstem/spinal cord. Here, we used a variety of preparations to determine the type of spinal sensory inputs responsible for this respiratory rhythm entrainment, and to establish the extent to which limb movement-activated feedback influences the medullary respiratory networks via direct or relayed ascending pathways. During in vivo overground locomotion, respiratory rhythm slowed and became coupled 1:1 with locomotion. In hindlimb-attached semi-isolated preparations, passive flexion-extension movements applied to a single hindlimb led to entrainment of fictive respiratory rhythmicity recorded in phrenic motoneurons, indicating that the recruitment of limb proprioceptive afferents could participate in the locomotor-respiratory coupling. Furthermore, in correspondence with the regionalization of spinal locomotor rhythmgenerating circuitry, the stimulation of DRs at different segmental levels in isolated preparations revealed that cervical and lumbosacral proprioceptive inputs are more effective in this entraining influence than thoracic afferent pathways. Finally, blocking spinal synaptic transmission and using a combination of electrophysiology, calcium imaging and specific brainstem lesioning indicated that the ascending entraining signals from the cervical or lumbar limb afferents are transmitted across first-order synapses, probably monosynaptic, in the spinal cord. They are then conveyed to the brainstem respiratory centers via a brainstem pontine relay located in the parabrachial/ Kölliker-Fuse nuclear complex.
\end{abstract}

\section{Introduction}

To accomplish vital homeostatic functions, respiratory rhythmogenesis must be capable of autonomous expression that is independent of all other motor actions. Nevertheless, under certain physiological conditions, breathing is susceptible to the influence of other rhythmic motor functions, such as vocalization (Manogue and Paton, 1982), or during swallowing (McFarland and Lund, 1995) and vomiting (Grélot et al., 1992) when respiration actually ceases. Similarly, rhythmic locomotor behavior, which occurs in parallel with respiration, is also able to influence ongoing breathing activity during physical exercise in many species, especially when accelerated displacement occurs. Here, the rate of respira-

Received Jan. 24, 2012; revised June 21, 2012; accepted July 10, 2012.

Author contributions: J.S. and D.M. designed research;A.G., M.L.B.-J., M.-J.C., and D.M. performed research; A.G. and M.L.B.-J. analyzed data; A.G., M.L.B.-J., J.S., and D.M. wrote the paper.

This work was supported by grants from the Conseil Régional d'Aquitaine. A.G. was a doctoral fellow of the Demain Debout organization. We thank Julien Bacque-Cazenave and Laura Cardoit for assistance with our in vitro experiments and histological controls, respectively.

*J.S. and D.M. contributed equally to this work.

Correspondence should be addressed to Didier Morin, Université Bordeaux Segalen, UMR CNRS 5287, Institut de Neurosciences Cognitives et Intégratives d'Aquitaine, 146 rue Léo Saignat, 33076 Bordeaux, France. E-mail: didier.morin@u-bordeaux1.fr.

M.-J. Cabirol's present address: Centre de Microscopie et d'Imagerie, Institut Fédératif de Recherche des Sciences Jean Roche, Faculté de Médecine Nord-Marseille, F-13916 Marseille, France.

DOI:10.1523/JNEUROSCI.0360-12.2012

Copyright $\odot 2012$ the authors $\quad 0270-6474 / 12 / 3211841-13 \$ 15.00 / 0$ tory movement increases and the two rhythms can even become coordinated on a 1:1 basis (Bramble and Carrier, 1983; Boggs, 2002). Although previous evidence suggested that the functional interaction between locomotor and respiratory behavior can be achieved by neurogenic (rather than biomechanical) processes arising from sensory signaling in species as diverse as birds (Funk et al., 1992; Boggs et al., 1997), dogs (Howard et al., 1969), cats (Iscoe and Polosa, 1976), and humans (Waisbren et al., 1990), the central nervous mechanisms and neuronal pathways underlying the coupling of these two otherwise independent motor systems is still poorly understood.

In this context, advances have been made in more recent studies using the isolated in vitro CNS of the newborn rat (Morin and Viala, 2002; Giraudin et al., 2008) and reduced in situ preparations of juveniles (Potts et al., 2005), where rhythmic activation of limb somatic afferents by direct electrical stimulation of cervical and lumbar dorsal roots (DRs) or forelimb flexor muscles, respectively, was found capable of phase resetting and entraining spontaneously generated respiratory rhythmicity with a 1:1 coordination. Furthermore, chemically inactivating the lateral parabrachial nucleus in the brainstem pontine region abolished the respiratory entraining effect of somatic afferent input, thereby implicating an intervening role for this structure in the locomotory-respiratory coupling process (Potts et al., 2005). To date however, the nature of the spinal 
sensory afferents responsible for respiratory entrainment as well as the ability of somatic inputs at different cord levels to exert entraining influences has been little investigated. Moreover, although there is evidence that long-ascending spinal projections are involved in mediating the somatic afferent-derived respiratory entrainment (Morin and Viala, 2002; Giraudin et al., 2008), whether the sensory information is conveyed to the pontine relay en route to the medullary respiratory centers via direct or polysynaptic spinal pathways has not been documented.

Here, in vivo locomotor and respiratory movements were recorded in newborn rats to establish that rhythm coupling does indeed occur in the freely behaving animal at early stages of postnatal development. Using semi-isolated CNS preparations with attached hindlimbs that were subjected to passive and active movements, we next characterized the type of sensory receptors involved in somatic afferent-respiratory entrainment. The ability of segmentally distributed spinal afferents to influence respiratory rhythmicity and the extent to which these ascending actions are relayed synaptically in the cord were then assessed using in vitro isolated brainstem/spinal cord preparations. Finally, combined electrophysiological, calcium imaging, and lesion experiments were conducted to confirm the essential mediating role played by the brainstem parabrachial/Kölliker-Fuse (PB/KF) complex in locomotory-respiratory coupling.

\section{Materials and Methods}

Experiments were conducted on 0- to 2-d-old Sprague Dawley rats from different litters that were obtained from timed pregnant female rats raised in our laboratory breeding colony. All experiments were performed in accordance with the local ethics committee of the Universite Bordeaux Segalen and the European Communities Council Directive.

In vivo experiments and EMG recordings. Animals were anesthetized by hypothermia until the loss of reflex responsiveness to tail pinch. Pairs of $50 \mu \mathrm{m}$ silver wires for electromyography (EMG) were then inserted into selected extensor limb muscles (triceps and gastrocnemius of the forelimbs and hindlimbs, respectively) and fixed with tissue glue (collodion; Sigma). Another pair of silver wires was placed into an intercostal muscle to record respiratory EMG activity. Animals were then warmed, and the temperature of the recording setup was maintained at $\sim 37^{\circ} \mathrm{C}$ by means of heating lamps.

To induce locomotion, a hollow cone filled with bedding material from the neonate's nest was positioned in front of the animal's muzzle (Jamon and Clarac, 1998). This apparatus, which exudes an olfactory stimulus, was then slowly pulled over a distance of $50 \mathrm{~cm}$ to elicit EMGrecorded episodes of locomotion.

In vitro isolated brainstem/spinal cord preparations and ventral root recordings. Animals were deeply anesthetized by hypothermia and decerebrated from the rostral end of the fifth cranial nerves. Using procedures identical to those described previously (Morin et al., 2000; Juvin et al., 2007), the skin and muscles were removed, and the preparations were then placed in a $100 \mathrm{ml}$ chamber filled with artificial CSF (aCSF) containing the following (in mM): $100 \mathrm{NaCl}, 4 \mathrm{KCl}, 1.2 \mathrm{NaH}_{2} \mathrm{PO}_{4}, 2 \mathrm{CaCl}_{2}$, $1.3 \mathrm{MgCl}_{2}, 25 \mathrm{NaHCO}_{3}$, and $30 \mathrm{D}$-glucose. This standard bathing solution was continuously equilibrated with $95 \% \mathrm{O}_{2} / 5 \% \mathrm{CO}_{2}, \mathrm{pH} 7.4$, and maintained at $10^{\circ} \mathrm{C}$ during the dissection. The brainstem and spinal cord with its dorsal and ventral roots still attached were isolated and the ensemble was then secured ventral surface upward to the Sylgard resin lining of a $10 \mathrm{ml}$ recording chamber. The bath temperature was progressively raised and thereafter maintained at $26^{\circ} \mathrm{C}$ by means of a Peltier system. A postdissection resting period of $30 \mathrm{~min}$ was systematically observed before recording procedures began.

Respiratory-related activity in spinal ventral roots and the phrenic nerves was recorded using glass suction electrodes. The signals were amplified $(\times 10,000)$ by differential AC amplifiers (AM System; Phymep), bandpass-filtered $(0.1-3 \mathrm{kHz})$, rectified, integrated ( $\tau=20 \mathrm{~ms}$; Neurolog System; Digitimer), digitized, and stored on a computer hard disk (using Spike 2 software; Cambridge Electronic Design) for off-line analysis.
Semi-isolated preparations. In a set of experiments, an in vitro preparation of the spinal cord and brainstem with intact hindlimbs still attached was used in which cervical ventral root or phrenic nerve recordings were used to monitor the respiratory network activity during sequences of imposed limb movements. The brainstem and rostral spinal cord were exposed as described above, and the preparation was then pinned down ventral side upward. Next, one or both hindlimbs were gently held by means of forceps that were moved forward and backward to passively mimic cyclic limb movements.

Electrical stimulations and electrolytic lesions. Using an 8-channel digital stimulator (A.M.P.I.), stimulus pulse trains $(0.2-2 \mathrm{~V}, 0.5 \mathrm{~ms}$ at $5-10$ $\mathrm{Hz}$ ) were applied to selected hindlimb muscles or dorsal spinal roots via a stimulating tungsten electrode (Frederick Haer) or a homemade glass suction electrode, respectively. DR stimulation followed the procedure described previously (Morin and Viala, 2002; Giraudin et al., 2008). Briefly, the threshold stimulus for so-called "low-threshold" (presumed proprioceptive) DR afferent fibers was determined by increasing the train shock intensity from a subthreshold value until a level at which an effect on the relative timing of the subsequent cycle of ongoing spontaneous respiratory rhythmicity was observed. Once the latter was established, and unless otherwise stated, this threshold stimulus intensity was applied to a given root throughout the course of the experiment.

In all our experiments involving the influences of repetitive DR stimulation, we defined entrainment as the cycle-to-cycle shortening of ongoing respiratory rhythmicity so that the latter's cycle period became matched (1:1 coupling) to that of the stimulus trains. The imposed entrainment frequency was selected on the basis of the spontaneous respiratory period. To this end, and according to detailed resetting and entraining procedures previously described (Morin and Viala, 2002), the mean control period in each preparation was first calculated for respiratory activity occurring immediately before DR stimulus onset. Thereafter, a suprathreshold DR train stimulus was applied at $50 \%$ of the mean control respiratory cycle to evoke a premature triggering (phase advance) of the next respiratory burst. Subsequently, a series of further DR stimulations (minimum of three trains) was delivered at a period that remained at half of the control respiratory cycle. As previously defined in the isolated neonatal rat preparation (Morin and Viala, 2002), full rhythm entrainment was indicated by a relatively constant latency between each stimulus and the elicited respiratory burst response.

To test the effectiveness of a modified aCSF containing elevated concentrations of $\mathrm{Ca}^{2+}$ and $\mathrm{Mg}^{2+}$ (see below) to differentiate between monosynaptic and polysynaptic spinal pathways, single suprathreshold stimulus pulses were applied to a selected DR and the solution's effects on local reflex pathways were assessed by recording motor responses in the ventral root of the same cord segment.

In a further series of experiments, a tungsten electrode (Frederick Haer;) was positioned within the pontine region of the brainstem to electrically stimulate (train stimulus pulses of $2-2.5 \mathrm{~V}, 0.5 \mathrm{~ms}, 10 \mathrm{~Hz}$ ) or to electrolytically destroy ( $4-5 \mathrm{~V}$ for $5 \mathrm{~s}$ ) the area containing the $\mathrm{PB} / \mathrm{KF}$ nuclei.

Calcium imaging. For calcium imaging experiments, isolated brainstem/spinal cord preparations (with the cerebellum removed) were incubated in the calcium indicator Oregon Green 488 Bapta-1 AM (Invitrogen; special packages). A solution of $50 \mu \mathrm{g}$ of the dye dissolved in $2 \mu \mathrm{l}$ of $10 \%$ pluronic acid and $25 \mu \mathrm{l}$ of dimethylsulfoxide was added directly to $3 \mathrm{ml}$ of aCSF in an incubation chamber containing the preparation and maintained in the dark at room temperature for $1 \mathrm{~h}$ under constant oxygenation. After loading and a recovery period of $30 \mathrm{~min}$, the whole hindbrain was positioned dorsal side up in the recording chamber. Imaging was performed with a spinning disk confocal microscope (Yokogawa CSU 10, Olympus BX51 WI, and an Andor EMCCD camera iXon-897) equipped with a $4 \times$ objective. The camera speed was typically five frames per second, and film sequences usually lasted from 1-3 min. The averaged fluorescence within contours of interest was read for each movie frame. The fluorescence change over time was defined as $\Delta F / F=\left(F-F_{\text {basal }}\right) / F_{\text {basal }}$, where $F$ is the fluorescence at any point, and $F_{\text {basal }}$ represents the averaged baseline fluorescence throughout a given film sequence. The movies were analyzed using open-source 


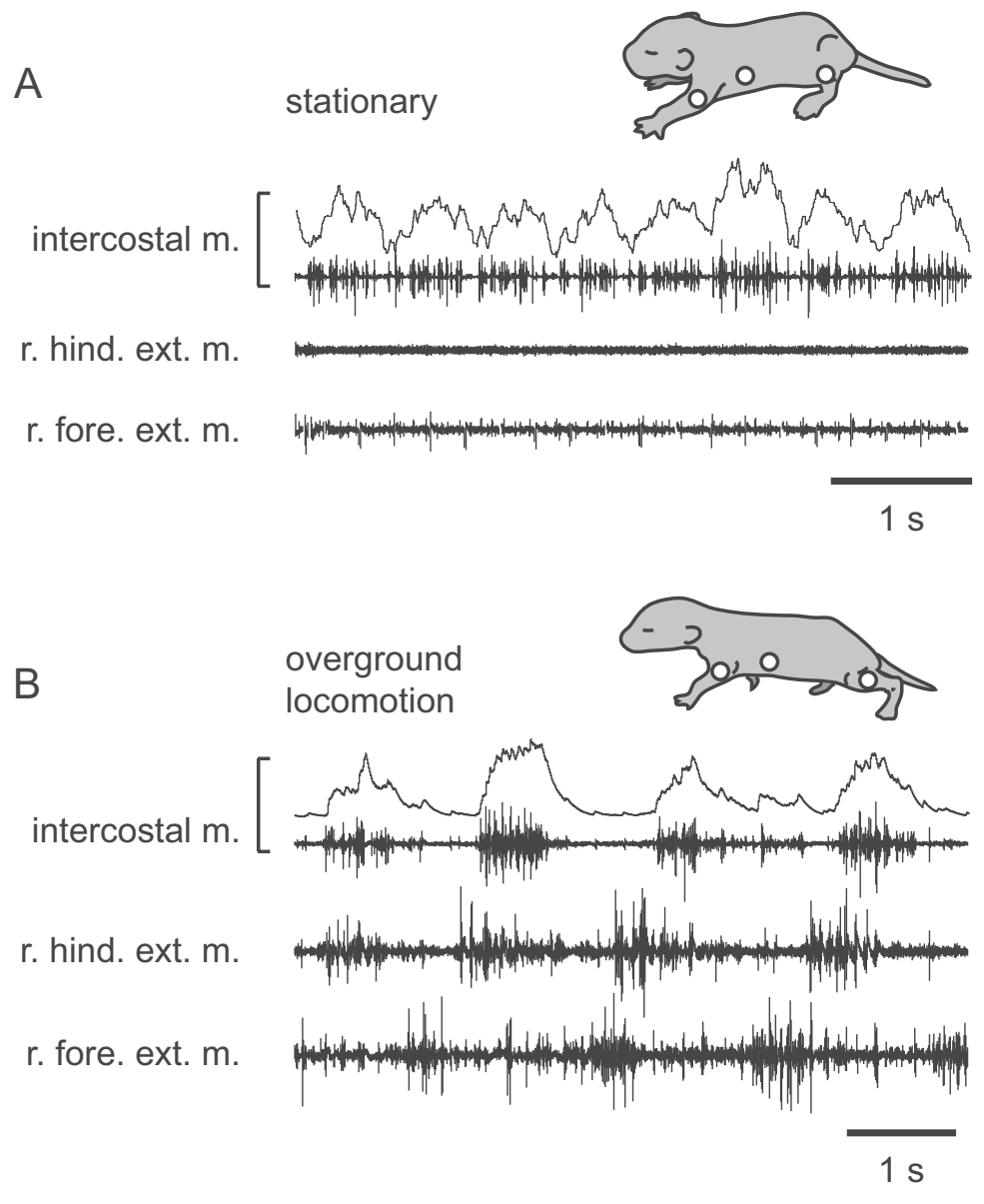

Figure 1. Locomotor-respiratory coupling in the neonatal rat during overground locomotion. Electromyographic recordings of a respiratory intercostal muscle (intercostal $\mathrm{m}$.), and right (r.) hindlimb (hind.) and forelimb (fore.) extensor (ext.) muscles at rest $(\boldsymbol{A})$ and during an olfactory stimulus-induced episode of locomotion in the same animal $(\boldsymbol{B})$. The integrated transform of the intercostal EMG activity is shown in the top trace of each recording panel. Sites of limb and intercostal EMG recordings are indicated (open circles) on the animal schematics.

software (ImageJ; National Institutes of Health) and IQ software (Andor Technology).

Modified saline applications. To characterize how sensory afferent information is relayed in the spinal cord, the recording chamber was partitioned into two compartments with a narrow barrier of syringeejected Vaseline placed transversally at the high cervical level (segment $\mathrm{C} 1$ ). To reversibly suppress polysynaptic transmission in the spinal cord, an aCSF enriched with $\mathrm{Ca}^{2+}(4 \mathrm{~mm})$ and $\mathrm{Mg}^{2+}(6 \mathrm{~mm})$ was then selectively applied to the spinal cord compartment. Water tightness was checked at the end of the experiment by sequentially adding methylene blue to the bathing medium on each side of the bridge. In other experiments, the spinal cord was exposed to a mod-

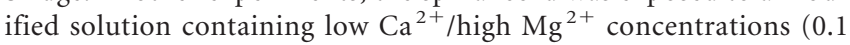
mu $\mathrm{CaCl}_{2}, 5 \mathrm{~mm} \mathrm{MgCl}_{2}$ ) to reversibly block all chemical synaptic transmission (Juvin et al., 2012) using similar bath-partitioning and verification procedures.

Brainstem transections and histological controls. In several isolated brainstem/spinal cord preparations, respiratory activity was recorded from a cervical ventral root while progressively more caudal transverse sections of the brainstem were made with a scalpel blade to locate any pontine structures that might be involved in spinal DR stimulationinduced respiratory entrainment. Recordings were made from $\sim 20 \mathrm{~min}$ after each transection to allow for recovery of the preparation.

To verify lesion locations in relation to the different brainstem nuclei, following recordings, preparations were fixed for $48 \mathrm{~h}$ at $4^{\circ} \mathrm{C}$ in Lillie solution (10\% formalin in phosphate buffer; $\mathrm{pH} 7.0)$. The tissue was then rinsed twice in phosphate buffer and cryoprotected overnight with $25 \%$ sucrose in phosphate buffer. After embedding in Tissue Tek and freezing with isopentane until $-80^{\circ} \mathrm{C}$, frozen $40-\mu \mathrm{m}$-thick serial parasagittal sections were cut on a cryostat. Adjacent sections were individually mounted on gelatincoated slides and alternately stained for acetylcholinesterase (AChE) or with cresyl violet to visualize the approximate boundaries of the pontine nuclei. For AChE staining, the slidemounted sections were dried at room temperature. They were then rinsed in $0.2 \mathrm{M}$ acetate buffer, pH 5.9, for $5 \mathrm{~min}$ before immersion in $0.2 \mathrm{M}$ acetate buffer containing $0.04 \mathrm{M}$ glycine and $0.01 \mathrm{M}$ copper sulfate pentahydrate for $18 \mathrm{~h}$ under agitation at room temperature. The sections were incubated in the same solution containing $1 \%$ acetylthiocholine iodide for 2 min under agitation at room temperature and rinsed three times in acetate buffer. They were then dehydrated in an ascending ethanol series, cleared in two changes of xylene, and mounted in Eukitt (Electron Microscopy Science) before observation under microscopy (DMRB; Leica).

Data analysis. The differences between means ( \pm SEM) were analyzed using a statistical software package (Sigma Stat for Windows; SPSS) and assessed either by a Student's $t$ test or one-way ANOVA with a Student-NewmanKeuls post-test. Differences in mean values were taken to be significant at $p<0.05$.

In a series of experiments, poststimulus time histograms (PSTHs) were computed to visualize the preferred delay times of ventral root respiratory discharge evoked by DR electrical stimulation. The PSTH gave the firing probability of a given ventral root in time bins of 1 and $2 \mathrm{~s}$ before, and $15 \mathrm{~s}$ after each stimulus pulse train (see Fig. 4F1). The preferred latency of the ventral root respiratory bursts triggered by spinal sensory afferent activation was assessed in terms of response probability (RP), which was calculated as the number of individual responses in the peak cohort divided by the total number of DR stimulations. Finally, a measure of the dispersion of the probability distribution in the PSTH was determined by calculating the mean coefficient of variation (Fig. 4F2), which was defined as the ratio of the SD to the mean.

\section{Results}

\section{Involvement of limb proprioceptive afferents in} locomotor-respiratory coupling

Muscle activity underlying respiratory and locomotor movements was initially monitored in behaving neonatal rats $(n=4)$ during olfactory stimulus-evoked episodes of locomotion (Jamon and Clarac, 1998; Juvin et al., 2005) to determine whether locomotor-respiratory coupling could occur in the freely moving animal. To this end, EMG recordings of homolateral forelimb elbow (triceps) and hindlimb ankle (gastrocnemius) extensor muscles were made simultaneously with an intercostal muscle to monitor the inspiratory phase of each respiratory cycle. At rest, animals expressed rapid breathing movements that were characterized by short inspiratory cycle periods (ranging from 450 to $600 \mathrm{~ms}$; Fig. 1A) as previously reported (Hilaire et al., 1993). During overground locomotion, a close coordination between stepping and breathing patterns was observed (Fig. $1 B$ ) in two of four animals, with the establishment of a 1:1 coupling similar to 
that observed among a variety of other quadruped species (Boggs, 2002). When such coupling occurred in our in vivo experiments, moreover, the frequency of inspiratory bursts decreased to become time locked to the locomotor rhythm, strongly suggesting that the latter was imposing its periodicity on the former.

In isolated brainstem/spinal cord preparations of the neonatal rat, we previously reported that phasic electrical stimulation of lumbar (Morin and Viala, 2002) or cervical (Giraudin et al., 2008) DRs in a manner that mimics locomotion-induced activation of somatosensory afferents in vivo can reset and entrain respiratory rhythmicity, indicating that these spinal inputs have access to the brainstem respiratory centers. Moreover, since only low stimulus intensities were required to activate the lumbar and cervical sensory pathways (Morin and Viala, 2002; Giraudin et al., 2008; also see below) it was postulated that larger diameter fibers (most likely proprioceptive and cutaneous afferents) were responsible for the DR stimulation-evoked influences on respiratory rhythmogenesis.

To confirm the proprioceptive origin of these spinal inputs, we used hindlimbattached preparations $(n=8)$ in which fictive respiration was monitored in a phrenic nerve of the rostrally isolated CNS while passive rhythmic movements were imposed on the still-intact hindlimbs. The applied limb motion approximated the movement excursions and cycle periods occurring during actual locomotion and therefore was likely to preferentially activate proprioceptive afferents that would provide phasic sensory feedback to the lumbar spinal cord during walking movements (Clarac et al., 2000; Duysens et al., 2000; Pearson, 2000).

In a first set of experiments, successive passive flexions and extensions that were alternately applied to both hindlimbs resulted in an $80 \%$ increase in the respiratory rhythm frequency in two of the four semi-isolated preparations examined (Fig. 2A). Moreover, in four other intact hindlimb preparations, repetitive flexion-extension movements applied to a single hindlimb consistently modulated ongoing respiratory rhythmicity by shortening its cycle period (Fig. $2 B$ ). These results therefore show that movement-induced activation of hindlimb sensory receptors, whether unilaterally or bilaterally, can effectively govern the cycle rate of respiratory activity.

In a further series of experiments $(n=6)$, we used similar semi-isolated preparations in which the skin had been entirely removed from the still-attached hindlimbs. In addition to avoiding the possible involvement of cutaneous mechanoreceptor afferent activation in the entraining process, this procedure allowed electrical train stimulation to be directly applied to individual identified muscles (Table 1) to produce limb movements through active muscle contractions. Similar to the results obtained during passive unilateral limb movements (Fig. $2 \mathrm{~B}$ ), a full $1: 1$ entrainment of respiratory rhythmicity recorded in cervical
Table 1. Proportions of hindlimb-attached brainstem/spinal cord preparations displaying respiratory rhythm entrainment in response to electrical stimulation-induced contractions of different limb muscles

\begin{tabular}{lcc}
\hline Stimulated muscle & $n$ & Respiratory entrainment \\
\hline $\begin{array}{l}\text { Hamstrings } \\
\quad \text { Knee flexor }\end{array}$ & 5 & $100 \%(5 / 5)$ \\
$\begin{array}{l}\text { Quadriceps } \\
\quad \text { Knee extensor }\end{array}$ & 4 & $25 \%(1 / 4)$ \\
$\begin{array}{l}\text { Tibialis anterior } \\
\text { Ankle flexor }\end{array}$ & 2 & $100 \%(2 / 2)$ \\
$\begin{array}{l}\text { Gastrocnemius } \\
\text { Ankle extensor }\end{array}$ & 5 & $80 \%(4 / 5)$ \\
\hline
\end{tabular}

motoneurons was elicited by electrical stimulation-evoked hindlimb muscle contractions (Fig. 2C), resulting in a consistent and significant decrease in respiratory period from $25.8 \pm 1.5 \mathrm{~s}$ (mean control value) to $12.1 \pm 0.6 \mathrm{~s}(p<0.001, n=5$ preparations) during muscle stimulation. However, this entraining ability was significantly stronger with flexor rather than extensor muscle activation, and in particular, the muscles controlling knee flexion (Table 1). Moreover, no effects on respiratory activity 
<smiles></smiles>
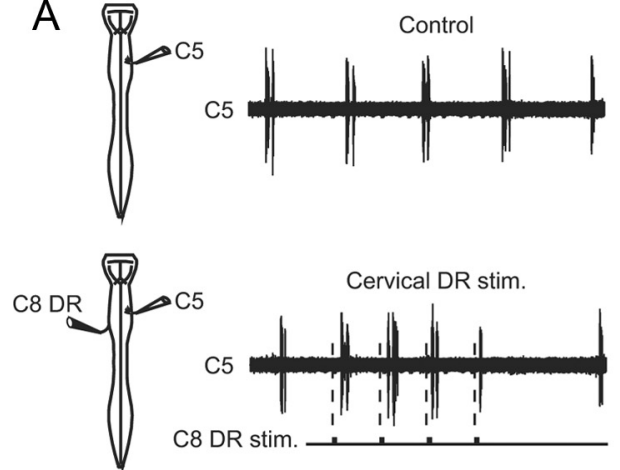<smiles>CC(=O)c1ccccc1Br</smiles>
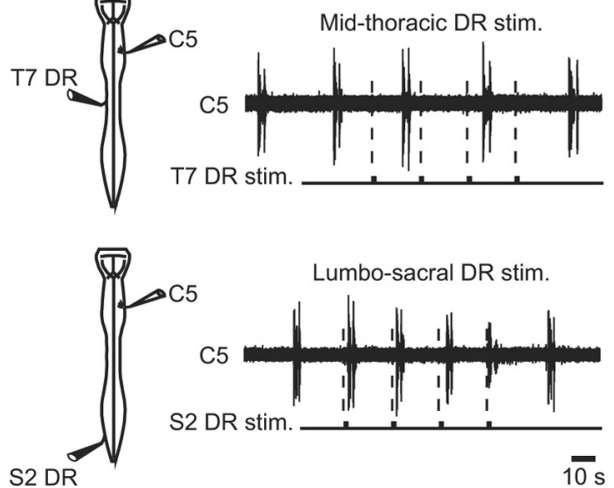

B1

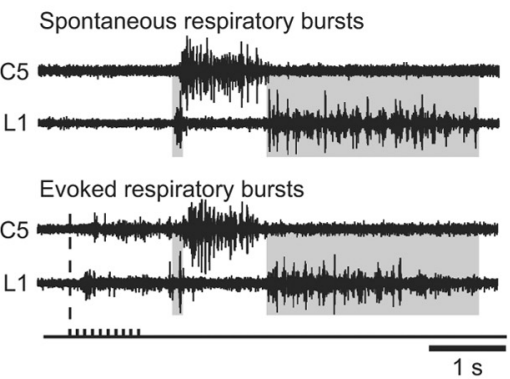

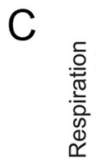
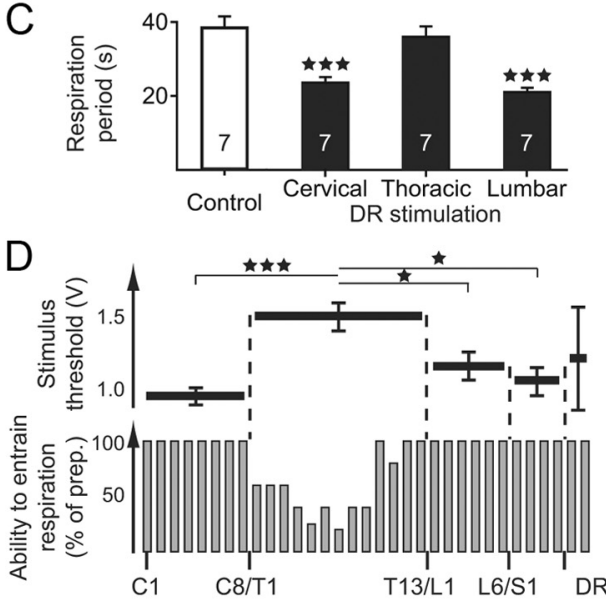

Figure 3. Respiratory rhythm entrainment by the cyclic activation of spinal low-threshold somatic afferent pathways. $A$, Left, Schematics of isolated brainstem/spinal cord preparations showing electrode placements for extracellular ventral root recordings and DR stimulation in each experimental condition. Right, Cervical (C5) ventral root activity under control conditions and during repetitive electrical stimulation of a cervical (C8), thoracic (T7), or sacral (S2) DR (DR stim., bottom traces). Respiratory rhythm entrainment occurred during cervical or lumbosacral DR stimulation, but not with thoracic DRactivation. $\boldsymbol{B}$, Expanded traces of spontaneous inspiratory $(\mathrm{C} 5)$ and expiratory (L1) bursts (B1) and of an equivalent respiratory cycle elicited by a single $\mathrm{C} 8 \mathrm{DR}$ train stimulation (B2). C, Histograms of the mean respiratory cycle period (vertical bars) and corresponding SEM (vertical lines) in control condition (unfilled bar) and during cyclic train stimulation of a cervical, thoracic, or lumbosacral DR (filled bars). Data were pooled from seven preparations in each case. ${ }^{* * *} p<0.001$. D, Top, Horizontal bar plots indicating mean levels of stimulus threshold intensity for respiratory entrainment by individual DRs along the spinal cord. Vertical lines: SEM; ${ }^{*} p<0.05 ;{ }^{* * *} p<0.001$. Bottom, Histograms (expressed as percentage of preparations) indicating the ability of the DR afferents to entrain spontaneous fictive respiration. C, cervical; $T$, thoracic; L, lumbar; $S$, sacral.

were observed when the stimulating electrode was either implanted in muscles not associated with the limbs or simply placed elsewhere in the bathing solution of the experimental chamber. Here again, these data further confirm that the coupling between passive or active limb movements and the respiratory rhythm generator derives from sensory receptor signaling by limb somatic proprioceptors.

\section{Regional specificity of somatosensory influences and spinal relay pathways}

Distributed inputs from muscle proprioceptor afferents to the spinal cord constitute an already functional sensory system in the newborn rat (Kudo and Yamada, 1987) that is known to be activated during actual locomotion (Clarac et al., 2000; Duysens et al., 2000; Pearson, 2000). During self-motion, however, the forelimb, hindlimb, and trunk muscles are activated differently in terms of timing, amplitude of motor drive, and strength of contraction (Falgairolle et al., 2006). A reasonable prediction, therefore, is that the proprioceptive influences arising from these diverse muscle populations do not contribute in an equivalent manner to the process of respiratory rhythm entrainment.

To assess the capacity of spinal proprioceptive afferents of different origins to influence respiratory rhythmicity, repetitive low-intensity electrical stimulation ( $1 \mathrm{~s}$ pulse trains of $0.5-1.2 \mathrm{~V}$ at $10 \mathrm{~Hz}$; see Materials and Methods) to preferentially recruit large-diameter, presumed proprioceptive, fibers (Morin and Viala, 2002; Juvin et al., 2012) were applied in turn to cervical, thoracic, lumbar, sacral, or coccygeal DRs in isolated brainstem/ spinal cord preparations $(n=20)$. Spontaneous inspiratory and expiratory bursting activity was monitored simultaneously from C5 (phrenic) and L1 (abdominal) ventral roots, respectively. Under these conditions, the cyclic activation of low-threshold cervical [mean stimulation threshold (T) $0.94 \pm 0.06 \mathrm{~V}$; Figure 3D, upper bar plot], lumbar (T $1.14 \pm 0.10 \mathrm{~V})$, sacral (T $1.05 \pm 0.10$ $\mathrm{V})$, or coccygeal (T $1.20 \pm 0.34 \mathrm{~V}$ ) somatic afferents was able to entrain respiratory bursting in a 1:1 coupling in all preparations tested ( $n=5$ for each DR type; Fig. $3 A, C, D$, lower). As previously reported for lumbar and cervical DR stimulation (Morin and Viala, 2002; Potts et al., 2005; Giraudin et al., 2008), this rhythm entrainment derived from the ability of spinal somatic afferent activation to repetitively reset the timing of bursts produced by the brainstem respiratory centers. Consistent with this cycle-bycycle resetting effect, no differences were evident in the temporal structure of spontaneously produced (Fig. 3B1) and DR-evoked respiratory burst patterns (Fig. 3B2), which in both cases typically consisted of double expiratory bursts in L1 motoneurons alternating with a single inspiratory burst in the $\mathrm{C} 5$ ventral root per cycle. In contrast, the capacity to entrain ongoing respiratory rhythmicity was significantly weaker when thoracic DRs were stimulated, especially those between T4 and T9 (Fig. 3A, C,D, lower), and even with a 1.5- to 2 -fold increase (mean $1.50 \pm$ $0.10 \mathrm{~V}$ ) in stimulus strength (Fig. $3 D$, upper). Together these findings therefore indicate that the effectiveness of spinal proprioceptive afferents in driving respiratory periodicity was in- 
A

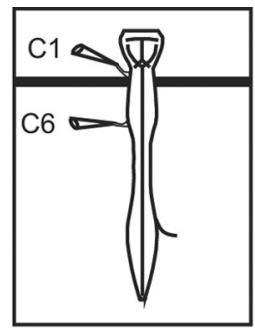

B

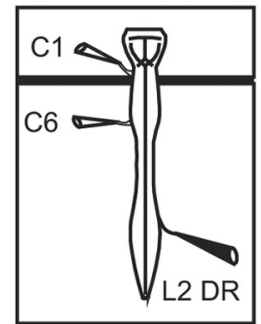

C

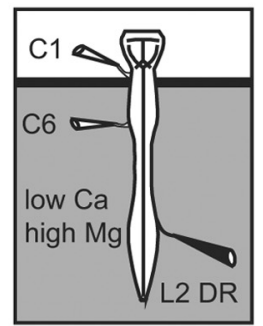

D

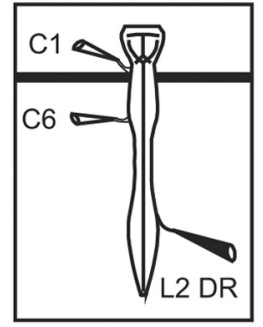

$\mathrm{E}$
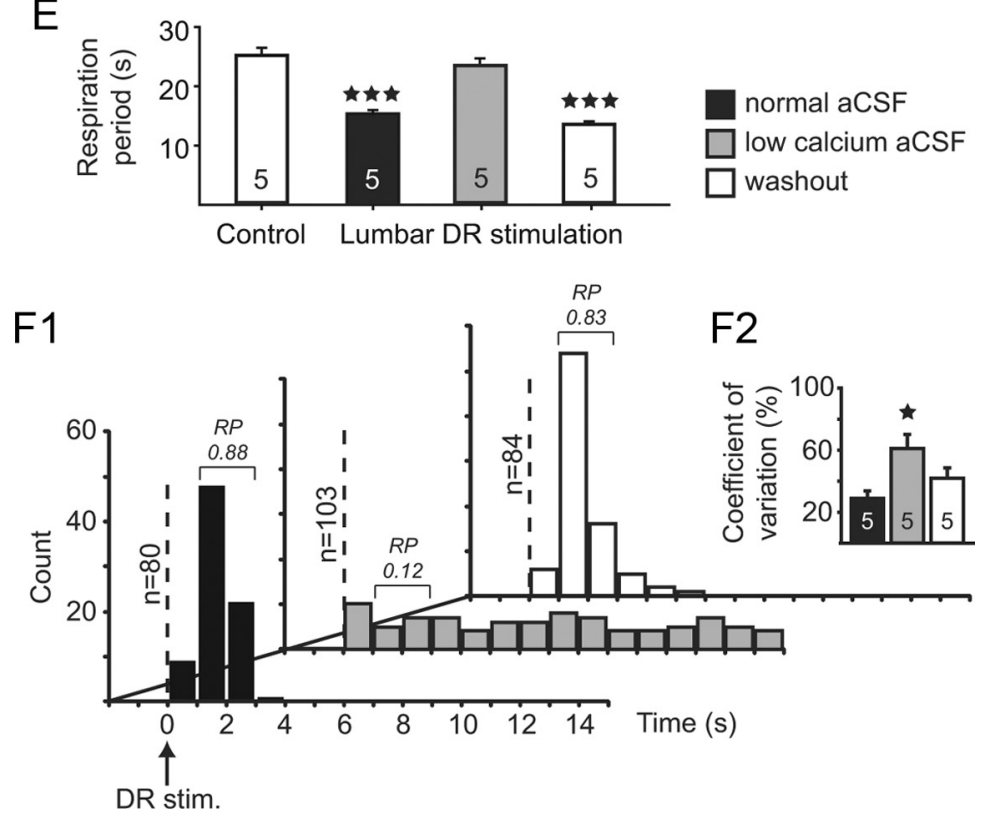

Figure 4. The influence of somatic afferent signaling on medullary respiratory circuitry is mediated by a spinal synaptic relay. $\boldsymbol{A}-\boldsymbol{D}$, Left, Schematics of experimental procedures. In each case, the horizontal black line represents the position of a Vaseline deed regionalized along the cord, with a diminished influence of somatic afferent inputs that would normally arise from the trunk musculature.

In a next step, spinal cords were subjected to modified aCSF applications to determine whether the DR-evoked respiratory entrainment was mediated by direct or synaptically relayed ascending pathways. First, to determine the contribution of local intersegmental connectivity to this coupling, chemical synaptic transmission in the spinal cord was reversibly blocked by selectively exposing it to low $\mathrm{Ca}^{2+} / \mathrm{high} \mathrm{Mg}^{2+}$ medium $(n=5)$ (Juvin et al., 2012). For this, a thin barrier of Vaseline was positioned just caudally to the $\mathrm{C} 1$ spinal segment to allow the brainstem respiratory centers to remain active under normal aCSF perfusion conditions (Fig. 4). As shown previously, during application of normal aCSF to both the brainstem and spinal cord, cyclic train stimulation of low-threshold cervical (C7 or C8, $n=5$ ) or lumbar (L2 or L4, $n=5$ ) DR afferents elicited 1:1 entrainment of respiratory-related bursting monitored on either side of the Vaseline barrier from $\mathrm{C} 1$ and C6 ventral roots (Fig. 4B,E). Moreover, as illustrated by the PSTHs in Fig. 4F1 (black bars), the onsets of these control DR stimulation-evoked respiratory bursts occurred at latencies of $1-3 \mathrm{~s}$ (mean, $1.62 \pm 0.06 \mathrm{~s} ; n=80$ ), with a pronounced RP $(0.88)$ and a relatively narrow temporal dispersion (coefficient of varia-

\section{$\leftarrow$}

barrier that allowed the cord to be selectively superfused with modified aCSF. A, Middle, Extracellular recordings of spontaneous respiratory activity in cervical ( $C 1$ and $(6)$ ventral roots under control conditions. $\boldsymbol{B}-\boldsymbol{D}$, The entrainment of respiratory bursting in $C 1$ and $(6$ by lumbar DR afferent stimulation (L2 DR stim.) under normal saline $(\boldsymbol{B})$ disappeared in the presence of

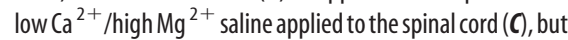
was restored after washout with normal saline (D). E, Histograms of mean respiratory period before (control, left unfilled bar) and during repetitive lumbar DR stimulation at half control period under normal saline perfusion (filled bar), during exposure of the whole spinal cord to a low $\mathrm{Ca}^{2+} / \mathrm{high}_{\mathrm{Mg}^{2+}}$ saline (gray bar), and after washout with normal saline (right unfilled bar). Data were pooled from five preparations. Vertical lines: SEM; ${ }^{* * *} p<0.001$. $F 1$, PSTHs of the delay of onset of subsequent ventral root respiratory burst discharge in response to single DR stimulations under normal saline (filled bars), modified divalent saline (gray bars), and after washout (unfilled bars). The number of DR stimulus trials is indicated (at left) for each case. Note that the flattened and dispersed PSTHs in low $\mathrm{Ca}^{2+}$ aCSF indicated a loss of DR stimulus-respiratory burst causality due to the blockade of spinal synaptic transmission. RP, response probability. F2, Histograms showing mean coefficients of variation (vertical bars) and SDs (vertical lines) under the three experimental conditions. The number of preparations is indicated on each bar. ${ }^{*} p<0.05$. 


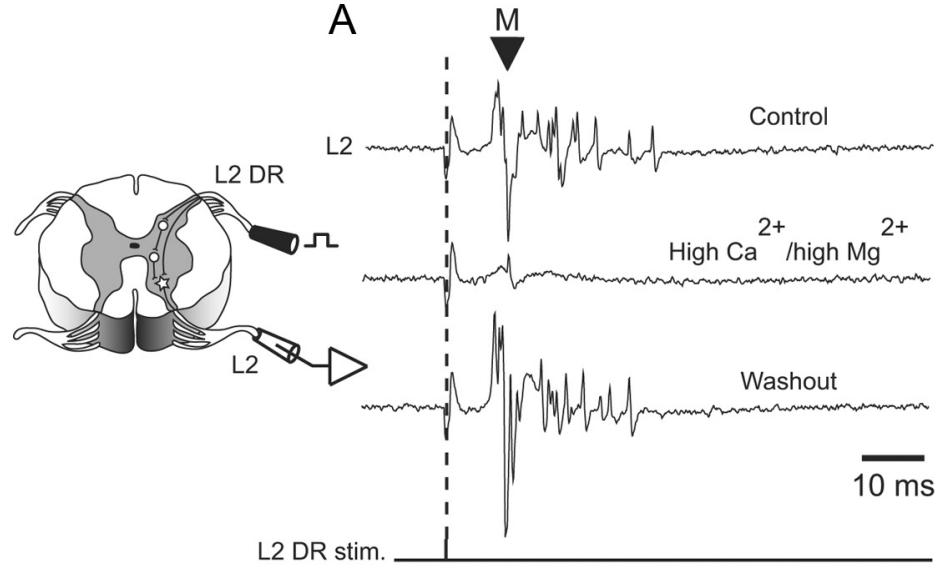

B

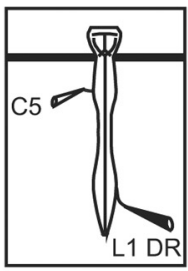

L1 DR stim
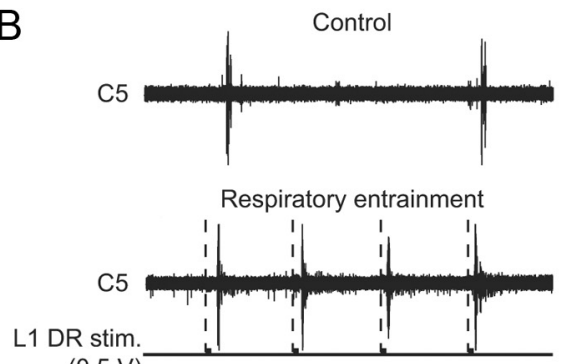

$(0.5 \mathrm{~V})$

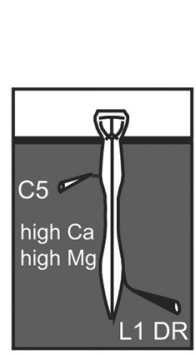

C1
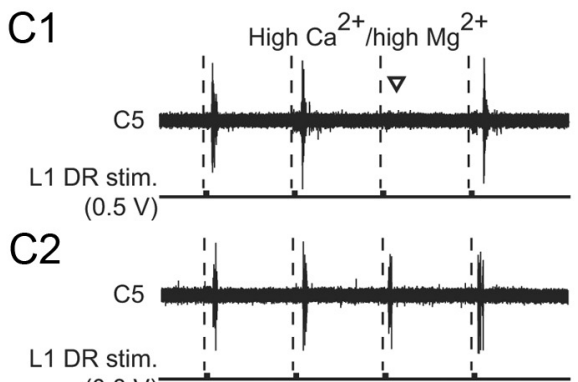

$(0.8 \mathrm{~V})$
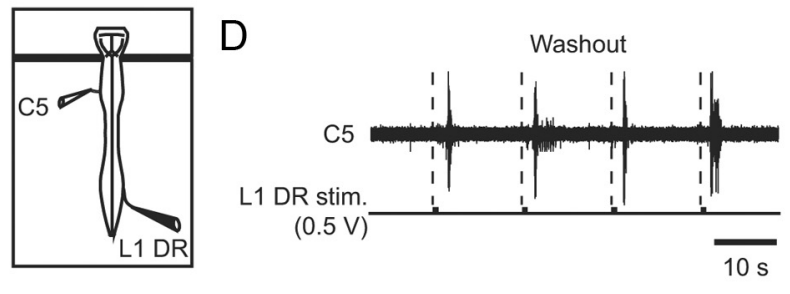

E

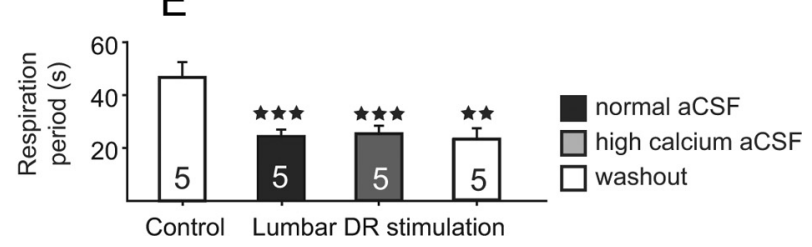

Figure 5. Evidence for the involvement of a paucisynaptic spinal relay in the respiratory rhythm entrainment by low-threshold DR afferent stimulation. $\boldsymbol{A}$, Ability of high divalent cation aCSF to suppress spinal polysynaptic pathways. Left, Schematic of the spinal cord in cross section and the segmental reflex pathways activated by low-threshold sensory afferent activation. DR, dorsal root. Right, L2 ventral root responses to single L2 DR stimulations (lower traces) in control conditions (top), during high $\mathrm{Ca}^{2+} /$ high $\mathrm{Mg}^{2+}$ application to the cord (middle) and after washout with normal aCSF (bottom). The enriched divalent solution reversibly blocked the long-latency (polysynaptic) activation of motoneurons without suppressing the short-latency (presumed monosynaptic, M) motor response (arrowhead). $\boldsymbol{B}-\boldsymbol{D}$, Left, Schematics of the isolated preparations and experimental procedures. A Vaseline barrier (indicated by horizontal bars) placed at the brainstem/spinal cord junction allowed differential aCSF bathing of the spinal cord. Middle, Effects of repetitive electrical stimulation of a lumbar dorsal root (L1 DR stim.) on respiratory motor bursting recorded

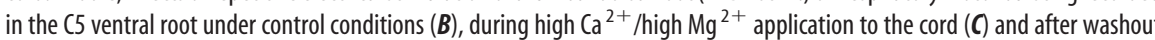

tion, $28.9 \pm 4.7 \%$; $n=5$; Fig. 4 F2). When the cord was now selectively exposed to a low $\mathrm{Ca}^{2+}$ solution, all spinal motor output was abolished, as was the ability of DR stimulation to entrain spontaneous respiratory bursting (here monitored more rostrally at $\mathrm{C} 1$; Fig. $4 C, E$ ), even when higher voltage stimulation intensities (up to $3 \times$ control $\mathrm{T}$ ) were applied. Accordingly, PSTH analysis confirmed the lack of any temporal relationship between each DR stimulation and the next spontaneous respiratory burst occurrence (Fig. 4F1, shaded bars), and as a consequence, the mean value of coefficient of variation was significantly increased $(61.0 \pm 9.0 \% ; n=5$; Fig. 4F2). These effects of low-Ca ${ }^{2+}$ aCSF, which were fully reversible (Fig. $4 D, E, F 1, F 2$, unfilled bars), thus strongly support the conclusion that the ascending signals responsible for the cervical or lumbar somatic afferent-induced entrainment of respiratory bursting are relayed indirectly via synaptic transmission within the spinal cord.

In a complementary series of experiments, a high $\mathrm{Ca}^{2+} /$ high $\mathrm{Mg}^{2+}$ aCSF was selectively applied to the spinal cord to assess the extent to which spinal synaptic relays intervene in conveying sensory information to the supraspinal respiratory centers. The ability of this modified solution to block spinal polysynaptic circuitry (Vinay et al., 1995) was initially verified on monosynaptic and polysynaptic reflex pathways within a given cord segment $(n=3)$. As seen in Fig. 5A (upper trace), in control conditions, a suprathreshold pulse stimulation of a lumbar (L2) DR elicited a short-latency motor response followed by a delayed, long-lasting discharge in the L2 ventral root. As previously reported (Juvin and Morin, 2005), the early response was consistent with the activation of a monosynaptic reflex pathway, whereas the long-lasting response corresponded to the parallel activation

$\leftarrow$

with a normal aCSF (D). The modified aCSF decreased, but did not abolish, the ability of lumbar afferent stimulation $(0.5 \mathrm{~V})$ to fully entrain ongoing respiratory rhythmicity $\left(C_{1}\right.$, open arrowhead indicates failed stimulus trial). However, a strict 1:1 coupling was restored when higher stimulus voltages (here $0.8 \mathrm{~V}$ ) were applied to the L1 DR (C2). E, Histograms showing changes in mean respiratory period before (control, unfilled bar) and during repetitive lumbar DR stimulations at half control period under normal saline superfusion (filled bar), during application of high $\mathrm{Ca}^{2+} /$ high $\mathrm{Mg}^{2+}$ (gray bar), and after washout with normal saline (right unfilled bar). Data were pooled from five preparations. Vertical bars, mean values; vertical lines, SEM; ${ }^{* *} p<0.01 ;{ }^{* * *} p<0.001$. 
A1
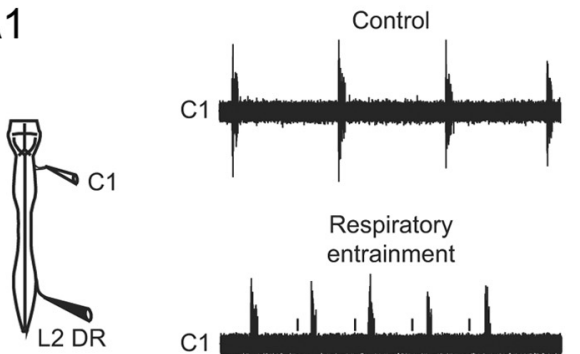

Respiratory entrainment

C1

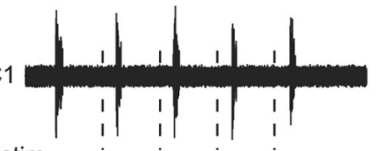

L2 DR stim

A2
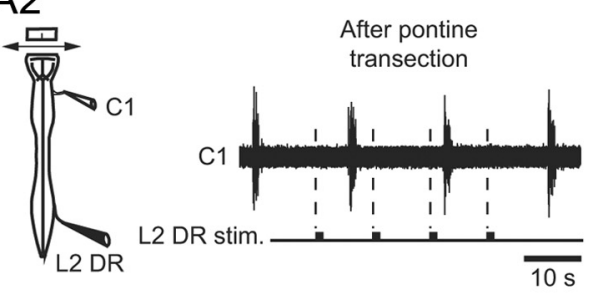

B

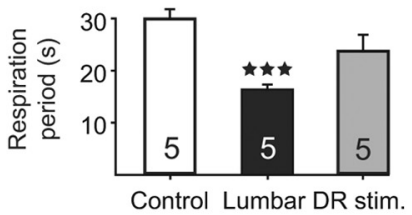

before pontine transection

after pontine transection
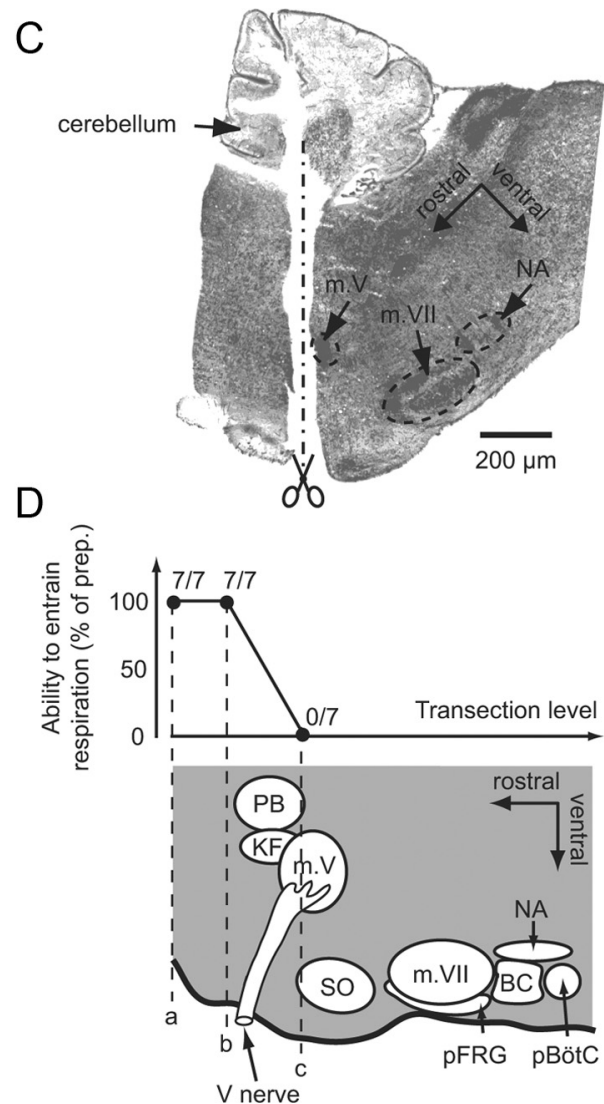

Figure 6. The pontine brainstem region is implicated in the pathway for respiratory rhythm entrainment by low-threshold spinal afferent activation. $\boldsymbol{A}$ 1, Spontaneous fictive respiration recorded in a C1 ventral root (top) and rhythm entrainment by cyclic L2 DR stimulation (lower) in a pontomedullary/spinal cord preparation. $\boldsymbol{A 2}$, Loss of entrainment after removal of the rostral pons. $\boldsymbol{B}$, Histograms of mean respiratory periods in control conditions (Control, unfilled bar) and during repetitive DR stimulation (lumbar DR stim., filled bars) at half control period before (filled bar) and after pontine transection (gray bar) in five preparations. Vertical lines, SEM; ${ }^{* * *} p<0.001$. C, Subsequent histological control of the pontine lesion (corresponding to level c in $\boldsymbol{D}$ ) in the preparation recorded in $\mathbf{A 2}$. The parasagittal section, which was stained for AChE, shows the transection (dotted line) located just rostral to the trigeminal motor nucleus (m. V) $\boldsymbol{D}$, Proportions of seven preparations expressing spinal DR stimulation-induced respiratory entrainment after successive brainstem transections at levels a-cindicated by the dotted lines in the parasagittal schematic of the pontine/medullary region. BC, Bötzinger complex; KF, Kölliker-Fuse nucleus; m. V, trigeminal motor nucleus; m. VII, facial motor nucleus; NA, nucleus ambiguous; pBötC, pre-Bötzinger complex; pFRG, parafacial respiratory group; $\mathrm{PB}$, parabrachial nucleus; $\mathrm{SO}$, superior olive; $\mathrm{V}$ nerve, trigeminal nerve.

of polysynaptic sensory-motor circuitry (Fig. $5 \mathrm{~A}$, left). When the spinal cord was superfused with a high $\mathrm{Ca}^{2+} / \mathrm{Mg}^{2+}$ medium, the delayed reflex discharge was reversibly suppressed whereas, although attenuated, the rapid motor response persisted (Fig. 5A, middle trace). This result therefore corroborates the earlier finding (Berry and Pentreath, 1976) that raising divalent concentrations in the preparation bathing solution raises spike threshold without significantly altering synaptic transmission, thereby increasing the likelihood of blocking (and thus revealing) polysynaptic connectivity.

On this basis, additional high $\mathrm{Ca}^{2+} / \mathrm{Mg}^{2+}$ experiments $(n=$ 7) were conducted to establish whether spinal DR stimulationinduced respiratory entrainment is relayed via oligosynaptic or polysynaptic connections within the spinal cord. Again, brainstem/spinal cord preparations were placed in a recording chamber partitioned in two compartments by Vaseline barrier positioned at the cervicomedullary junction. That low-threshold (T 0.4-0.5 V) lumbar or cervical afferent stimulation could fully entrain spontaneous respiratory activity was first verified by recording from an inspiratory $\mathrm{C} 5$ ventral root under control saline conditions (Fig. $5 B, E$ ). During high $\mathrm{Ca}^{2+} / \mathrm{Mg}^{2+}$ application to the spinal cord, cyclic L4 DR stimulation continued to alter ongoing respiratory rhythmicity. However, consistent with a decreased neuronal excitability under the elevated divalent aCSF conditions
(Fig. 5A, middle trace), a strict 1:1 coordination was now replaced by an intermittent coupling in which individual DR stimulationinduced respiratory bursts could occasionally fail to occur (Fig. $5 C 1$ ). Nevertheless, in these cases, higher stimulus voltages (from 0.6 to $0.8 \mathrm{~V}$ ) applied to the same lumbar (or cervical) DR could reestablish a full 1:1 respiratory entrainment (Fig. 5C2,E). Finally, 1:1 coupling with original control DR stimulus threshold intensities was restored after a return to normal aCSF bathing conditions (Fig. 5D,E). Therefore, together these data indicate that the pontomedullary respiratory centers receive limb proprioceptive inputs via an initial-stage relay in the spinal cord involving single (or at most very few in series) synaptic connections.

\section{A pontine relay mediates limb somatic afferent-respiratory coupling}

Although the above findings, in agreement with previous in vitro studies (Morin and Viala, 2002; Giraudin et al., 2008), clearly show that limb proprioceptive information has access to the respiratory CPG, whether the spinal sensory signals upon reaching the brainstem are conveyed directly to the respiratory rhythm generators or are transmitted indirectly via hindbrain relay circuitry (Potts at al., 2005) remains unknown in the neonatal animal. To address this issue, a first series of hindbrain lesions was conducted in vitro to assess the possible involvement of cerebellar 
A

Dorsally-exposed brainstem

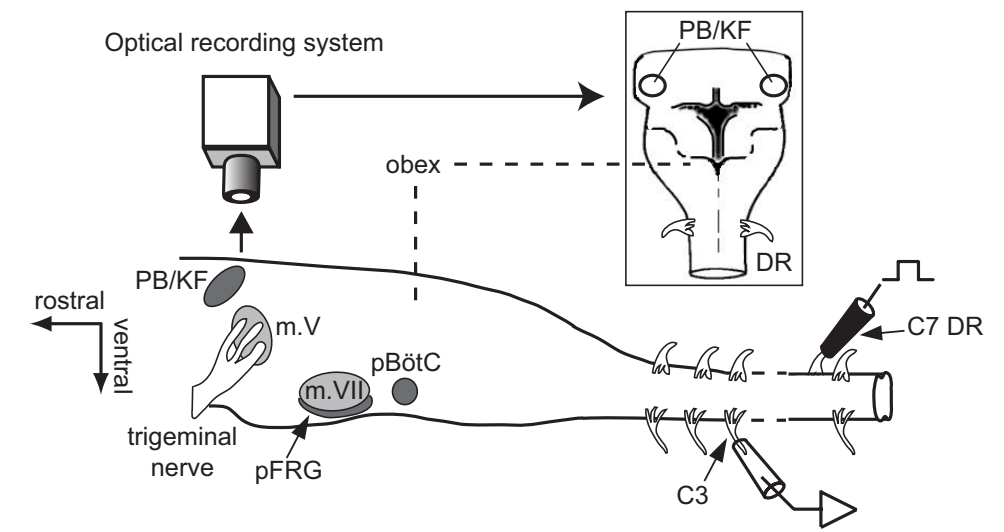

B
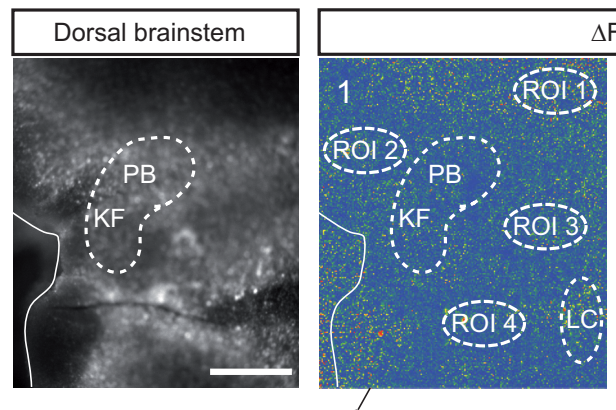

$\Delta \mathrm{F} / \mathrm{F}$

C

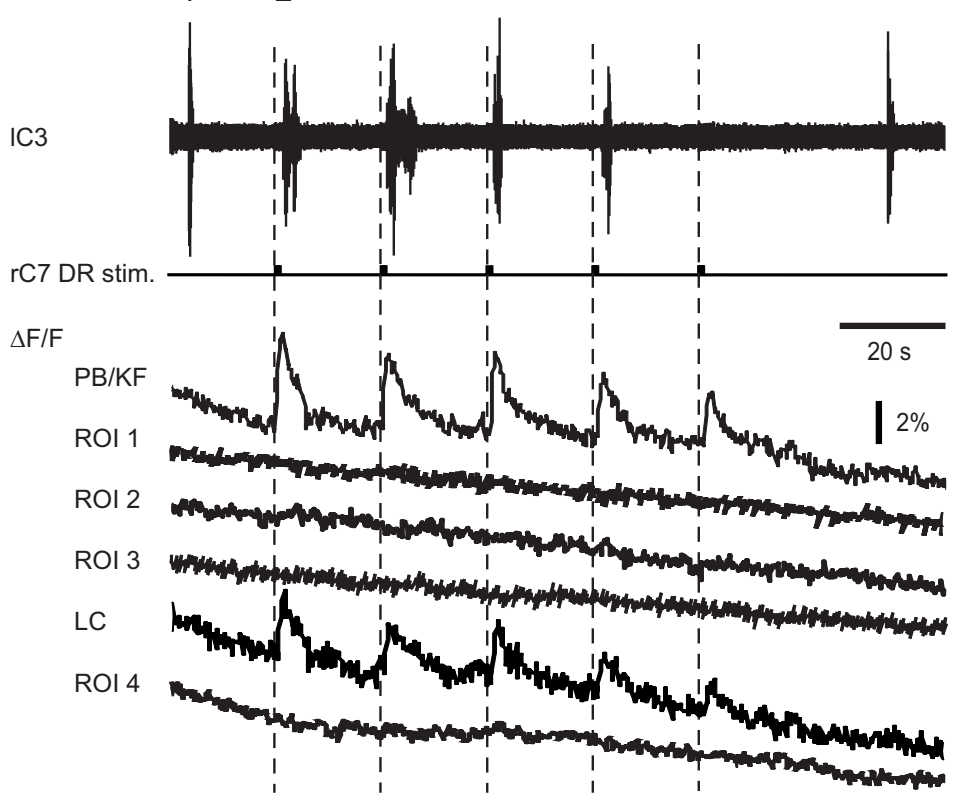

Figure 7. Activation of the dorsolateral pontine region during spinal DR stimulation-induced respiratory entrainment. $\boldsymbol{A}$, Schematic representation of experimental procedure using electrophysiology combined with calcium imaging. The inset shows a schematic dorsal view of the brainstem that was Oregon Green 1 AM loaded for optical recording. B, Left, Confocal image of the fluorescent $\mathrm{Ca}^{2+}$ indicator-loaded dorsal brainstem. Scale bar, $400 \mu \mathrm{m}$. Middle, Absence of fluorescence activity during a spontaneous respiratory burst (corresponding to 1 in C). Right, A transient fluorescence signal was expressed in the PB/KF pontine area (and with lesser intensity, a region corresponding to the locus ceruleus; LC) during a respiratory burst evoked by cervical C 7 dorsal root stimulation (see 2 in C). C, Simultaneous left C3 (IC3) ventral root recording of respiratory activity and associated fluorescence changes in the $\mathrm{PB} / \mathrm{KF}$ nuclei $\left(\triangle F / F_{\mathrm{PB} / \mathrm{KF}}\right)$ and the $\mathrm{LC}$, but not previously in four other neighboring regions (see $\left.\mathbf{B 1}, \mathbf{B 2}\right)$, during cyclic right C7 DR stimulation ( $\mathrm{rC} 7 \mathrm{DR}$ stim.). $\mathrm{m}$. V, trigeminal motor nucleus; $\mathrm{m}$. VIII, facial motor nucleus; $\mathrm{pBötC}$, pre-Bötzinger complex; pFRG, parafacial respiratory group; ROI, region of interest. structures, such as the dorsal spinocerebellar tract, which constitutes a major ascending pathway for transmitting limb proprioceptive information to the cerebellar cortex (for review, see Orlovsky et al., 1999). However, in all four in vitro preparations tested in the present study, a complete cerebellectomy did not modify the ability of spinal DR stimulation to entrain ongoing respiratory-related activity (data not shown), thereby indicating that the cerebellum itself does not intervene in the limb sensory-to-respiratory generator coupling.

In a second step, successively more caudal transections of the brainstem were made $(n=7)$ to establish whether pontine or medullary nuclei are involved in relaying spinal DR inputs to the respiratory centers. As seen in Fig. 6A1, transections made rostrally to the trigeminal $(\mathrm{V}) \mathrm{cra}$ nial nerves (Fig. 6D, levels a and b) did not affect the capacity of cervical or lumbar DR stimulation to entrain inspiratory bursts recorded in a $\mathrm{C} 1$ ventral root (Fig. $6 B)$. However, when a further transection was made more caudal to the $\mathrm{V}$ nerves (Fig. 6D, section c), DR stimulationevoked respiratory entrainment no longer occurred in all seven preparations examined (Fig. 6A2,B). A subsequent histological verification of the different lesion sites made with AChE staining of brainstem parasagittal sections (Fig. 6C), corroborated the physiological evidence that pontine structures located immediately rostral to the $\mathrm{V}$ nucleus are necessary components of a further relay intercalated between the ascending spinal pathway and the medullary respiratory centers.

In a third step, to further establish the importance of this pontine region to limb somatic afferent-respiratory coupling, we combined electrophysiological stimulation/recording with calcium imaging $(n=$ 9) to monitor associated neuronal activity in the brainstem. For this, the brainstem of in vitro preparations was first incubated en bloc with the Oregon Green Bapta 1-AM, then fluorescence changes due to somatic $\mathrm{Ca}^{2+}$ fluxes resulting from action potential generation (Yuste and Katz, 1991; Ikegaya et al., 2005) in the dorsal pontine region were monitored simultaneously with respiratory motor activity during low-threshold ( $\mathrm{T}$ 0.4-0.5 V) cervical or lumbar DR stimulation (Fig. 7A). $A$ cell population located within the $\mathrm{PB} / \mathrm{KF}$ complex (Fig. 7B, left) elicited distinct $\mathrm{Ca}^{2+}$ transients in response to individual DR stimulations (Fig. $7 B$, panel 2 in the average relative fluorescence signal $\left(\Delta F / F_{P B}\right)$ of Fig. $7 C$ and the corresponding 


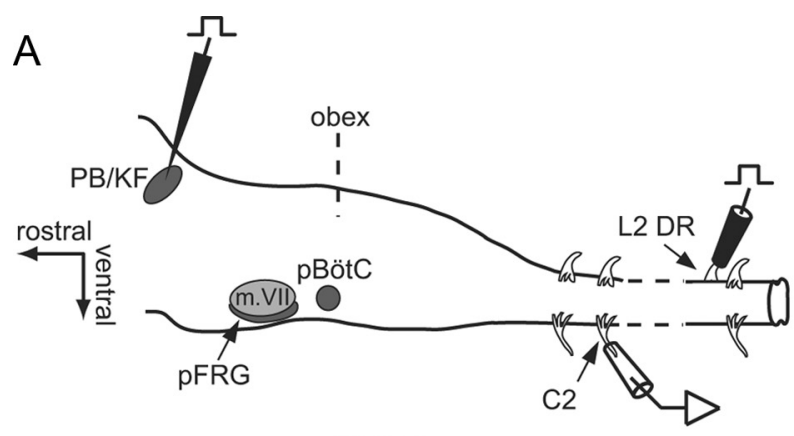

Control
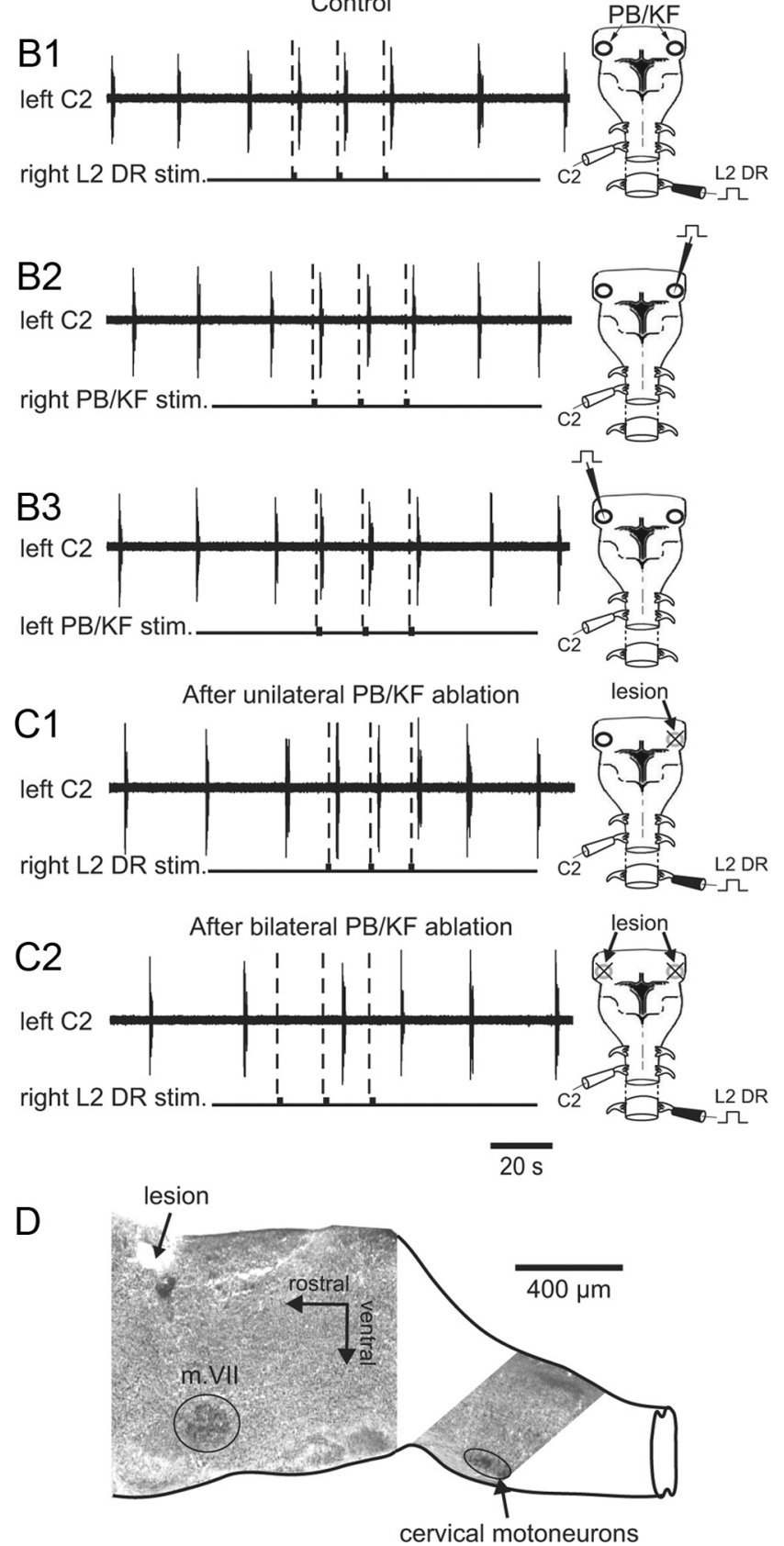

Figure 8. Spinal afferent-entrained respiratory bursting involves the $P B / K F$ complex as a pontine relay. $\boldsymbol{A}$, Schematic representation of brainstem structures (lateral view) and experimental procedures. $\boldsymbol{B}$, Right, Dorsal view diagrams of the brainstem/spinal cord preparation showing stimulating and recording electrode positions. Left, $\mathrm{C} 2$ ventral root recordings of inspiratory bursting during cyclic electrical stimulation of the contralateral L2 DR (B1) or during direct stimulation of the contralateral (right PB/KF stim., B2) or ipsilateral (left PB/KF stim., B3) PB/KF. Respiratory rhythm entrainment occurred with all three stimulus paradigms. $\boldsymbol{C}$, Persistence $(\mathbf{C} \mathbf{1})$ or suppression $(\mathbf{C})$ of respiratory optical response in Fig. $7 B$, right). These $\mathrm{PB} / \mathrm{KF}$-specific fluorescence changes were expressed with either cervical $(n=8)$ or lumbar $(n=4)$ DR stimulations, but significantly, they were not observed in the $\mathrm{PB} / \mathrm{KF}$ area when a spontaneous respiratory burst occurred (Fig. 7C, panel 1 and the corresponding optical response in Fig. $7 B$, middle). Moreover, in some instances with repeated DR stimulations, an optical response was evident in the $\mathrm{PB} / \mathrm{KF}$ complex without respiratory motor burst induction (Fig. $7 C$, last $\mathrm{Ca}^{2+}$ transient), here indicating a transmission failure of the limb afferent signal from the $\mathrm{PB} / \mathrm{KF}$ region to the respiratory generating centers. Presumably in such cases, the afferent-evoked excitation of $\mathrm{PB} / \mathrm{KF}$ neurons had progressively accommodated to a level that was subthreshold for respiratory generator activation. Finally, in addition to 1:1 suprathreshold responses observed in the $\mathrm{PB} / \mathrm{KF}$ complex, neurons located within the neighboring locus ceruleus displayed reliable signal coupling with spinal afferent stimulation (Fig. $7 B, C$ ), consistent with previous neuroanatomical evidence in rats that the $\mathrm{PB} / \mathrm{KF}$ also accesses the latter via communicating axonal projections (Luppi et al., 1995).

In a final set of in vitro experiments $(n=7)$, direct electrical stimulation and subsequent electrolytic lesions of the pontine brainstem region were made to further confirm the necessity of the $\mathrm{PB} / \mathrm{KF}$ complex to spinal limb afferent-respiratory CPG coupling. After removing the cerebellum and using the obex as an anatomical landmark, a stimulating tungsten electrode was placed in a presumed $\mathrm{PB} / \mathrm{KF}$ nuclear region of the dorsal pontine area, at a location $1.6 \pm$ $0.2 \mathrm{~mm}$ rostral to the obex and $1.0 \pm 0.1 \mathrm{~mm}$ from the midline, first ipsilaterally then contralaterally to the side of DR stimulation (Fig. $8 A$ ). Similar to the typical effects of lumbar or cervical DR stimulation observed under control conditions (Fig. 8B1), repetitive stimulus pulses delivered to either the ipsilateral (Fig. $8 B 2$ ) or contralateral $\mathrm{PB} / \mathrm{KF}$ (Fig. 8 B3) led to a 1:1 entrainment of ongoing respiratory rhythmicity. Although a unilateral electrolytic lesion in the region of the $\mathrm{PB} / \mathrm{KF}$ nuclei did not modify the capacity of spinal DR stimulation on either side of cord to entrain ongoing inspiratory bursting (Fig. 8C1), in six of the seven preparations tested, this entraining ability was irreversibly abolished by a subsequent bilateral $\mathrm{PB} / \mathrm{KF}$ ablation (Fig. 8C2). Moreover, histological procedures then verified that the lesion sites indeed corresponded to the location of $\mathrm{PB} / \mathrm{KF}$ nuclei in the dorsal region of the pons, at a depth of 150-200 $\mu$ m below the dorsal brainstem surface (Fig. $8 D$ ). Together these data therefore provide compelling evidence that in the neonatal rat, the $\mathrm{PB} / \mathrm{KF}$ complex serves as a necessary pontine conduit for the transmission of spinal proprioceptive afferent signals to respiratory rhythm-generating circuitry located more caudally in the ventral medulla.

\section{Discussion}

This study reports on the neural pathways underlying the functional coupling of rhythmic locomotor and respiratory pattern generation during locomotion in the neonatal rat. Observations in vivo and from semi-intact and isolated CNS preparations indicate that sensory inputs from somatic proprioceptive afferents activated by forelimb and hindlimb muscle contractions during rhythmic stepping movements are responsible for entraining ongoing respiratory rhythmicity. The ascending afferent information is relayed pauci-

$\leftarrow$

entrainment following unilateral (C1) or bilateral (C2) electrolytic lesions to the PB/KF nuclei, respectively. D, Parasagittal AChE-stained section as a histological control. The locality of the electrolytic lesion near the dorsal brainstem surface corresponded to the location of the PB/KF complex. m. VII, facial motor nucleus; pBötC, pre-Bötzinger complex; pFRG, parafacial respiratory group. 


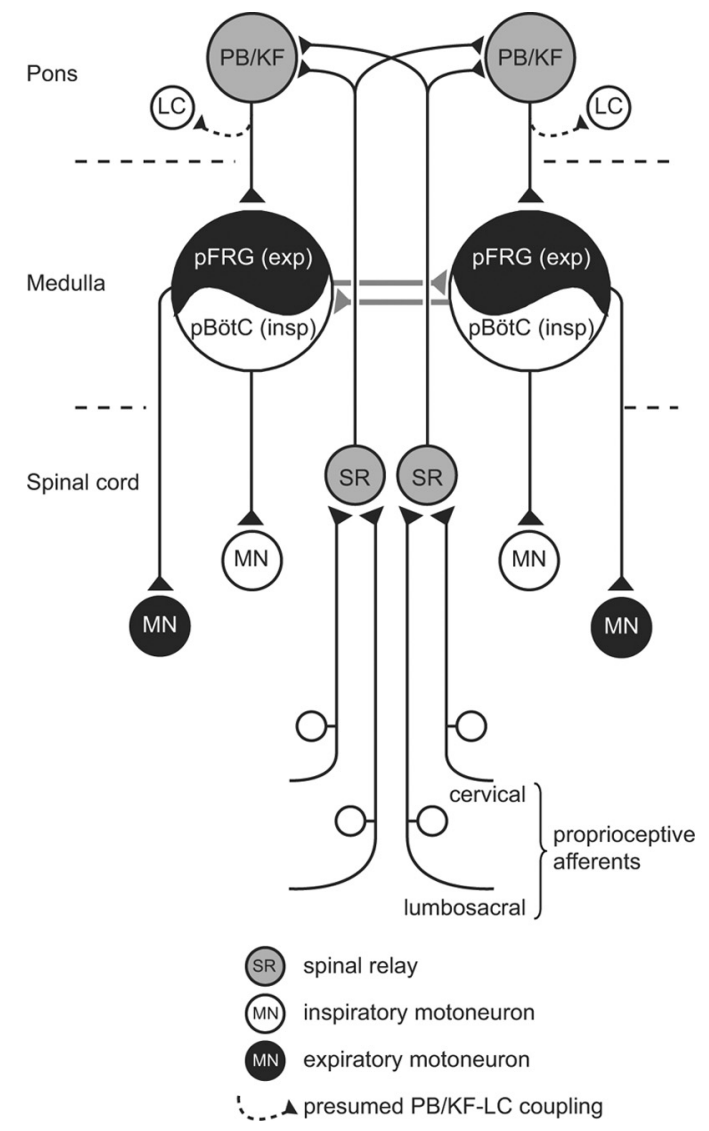

Figure 9. Summary diagram of the spinal and brainstem relay pathways involved in coupling respiratory rhythm generation to the rhythmic activation of cervical and lumbosacral proprioceptive inputs during locomotion. See text for further explanation. Exp, expiration; insp, inspiration; $\mathrm{pBötC,} \mathrm{pre-Bötzinger} \mathrm{complex;} \mathrm{pFRG,} \mathrm{parafacial} \mathrm{respiratory} \mathrm{group;} \mathrm{PB} / \mathrm{KF}$, parabrachial/Kölliker-Fuse nuclei; $L C$, locus ceruleus.

synpatically (and possibly) monosynaptically within the rostral spinal cord to bilateral second-order circuitry within the $\mathrm{PB} / \mathrm{KF}$ nuclear complex of the brainstem pontine region, from where it is conveyed to the medullary respiratory centers (Fig. 9).

\section{Locomotor-respiratory coupling in the neonatal rat}

Coordinating interactions between locomotor and respiratory rhythms are found in a wide range of animal species, including fish (Gariépy et al., 2012), birds, and mammals (for review, see Boggs, 2002), where it is thought to play a role in satisfying energetic demands. We report here for the first time that respiratorylocomotor coupling occurs during locomotion in the freely moving neonatal rat (Fig. 1), but in contrast to adult mammals, a $1: 1$ coordination is expressed at walking speeds that are substantially slower than the breathing frequency when the animal is stationary. In this case, therefore, the effective reduction (rather than increase) in respiratory rate during rhythm coupling does not readily comply with an energetic advantage.

One possibility is that the rhythm coordination is related to the immaturity of the newborn rat's spinal circuitry where, in the perinatal period, a proliferation of primary sensory afferents and their contacts with central postsynaptic targets occur, before these supernumerary connections decline in later postnatal ( $\mathrm{P} 4-$ P8) stages (Kudo and Yamada, 1987). Consequently, both the extent and central impact of somatic afferent recruitment during walking is likely to be greater in the newborn animal than the adult, which in turn would impart a higher probability of locomotor-respiratory coupling. It is also relevant here that the postnatal rat's ability to self-support and walk spontaneously remains limited until P10 (Westerga and Gramsbergen, 1990) due principally to the latent maturation of limb postural control (Brocard et al., 1999). In this still immature condition, therefore, the elevated physical effort required for body displacement may lead to an enhanced recruitment of muscle afferents.

It is also possible that the apparently paradoxical decrease in respiratory rate observed in the freely moving neonate is mainly biomechanical rather than neural in origin. Indeed, kinematic analysis in intact newborn rats has revealed a significant bending of the trunk axis during locomotion (Falgairolle and Cazalets, 2007). Given the highly compliant thoraxes of neonates, the lateral bending of their axial skeleton may constrain breathing during overground locomotion and consequently, as found in reptiles (Wang et al., 1997; Boggs, 2002), impose a slowing of respiratory frequency. On this basis, therefore, the respiratory entrainment at a reduced rate may reflect biomechanical rather than energetic constraints.

\section{Involvement of proprioceptive afferents in respiratory rhythm entrainment}

Consistent with previous in vivo data in dogs (Howard et al., 1969), cats (Iscoe and Polosa, 1976), rabbits (Palisses et al., 1988), and humans (Waisbren et al., 1990; Zhuang et al., 2009), in the neonatal rat, primary somatic afferent activation by passively imposed limb movements or by direct muscle stimulation can entrain respiratory rhythmicity in hindlimb-attached CNS preparations (Fig. 2). Moreover, in the isolated brainstem/spinal cord, respiratory entrainment occurred when spinal dorsal roots were rhythmically activated by low-threshold electrical stimulation (Fig. 3) to preferentially activate large-diameter sensory fibers (Morin and Viala, 2002; Juvin et al., 2012), thus further substantiating the involvement of proprioceptive pathways in the entraining process.

Perhaps predictably, and in agreement with previous studies (Morin and Viala, 2002; Potts et al., 2005; Giraudin et al., 2008), cyclic activation of cervical or lumbosacral DRs that normally arise from forelimb and hindlimb muscles, respectively, was found to be the most effective in entraining fictive respiration (Fig. 3). In contrast, repetitive stimulation of low-threshold thoracic (T4-T9) DR afferents failed to entrain respiratory activity in the majority of in vitro preparations. This reduced effectiveness is probably attributable to regional differences in the types of sensory inputs to rat DR ganglia, where proprioceptor fibers are almost 50\% less prevalent in thoracic DRs than cervical and lumbar ganglia (Chen et al., 1996). In some preparations, the diminished entraining ability of thoracic DR activation could be partially offset by increasing the strength of applied stimulation, which presumably led to the additional recruitment of smalldiameter group III and IV afferents that also have access to the respiratory generators (Persegol et al., 1993; Decherchi et al., 2007). Indeed in adult rats, the activation of metabosensitive group III and IV afferent fibers by electrically induced fatigue or in response to metabolic stimulation (by raising muscle potassium chloride or lactic acid concentrations) was found to significantly increase the rate of respiratory-related phrenic nerve activity (Decherchi et al., 2007).

Role of spinal and pontine relays in somatic afferent-induced respiratory entrainment

The application of low $\mathrm{Ca}^{2+}$ aCSF to the spinal cord revealed that cervical or lumbosacral DR activation is unable to produce respi- 
ratory rhythm entrainment in the absence of synaptic transmission within the cord sector of the ascending pathway (Fig. 4). Although the precise location of this first-order relay remains unknown, it evidently involves few synapses (and perhaps single connections) that are likely to be situated at the cervical cord level. This is compatible with earlier findings that a restricted blockade of synaptic transmission in lumbosacral (Morin and Viala, 2002) or thoracic (Giraudin et al., 2008) segments failed to suppress respiratory entrainment by spinal DR stimulation, indicating that these more caudal coupling signals are initially carried by long ascending projections.

A candidate site for the spinal relaying of somatic afferent information is the lateral cervical nucleus (LCN) located in the dorsal portion of the lateral funiculus of the C1-C3 segments. Although previous studies reported that LCN neurons are preferentially sensitive to hair movements and innocuous or noxious mechanical stimulation of the forelimbs and hindlimbs (Craig and Tapper, 1978; Metherate et al., 1986; Kajander and Giesler, 1987; Djouhri et al., 1994), LCN cells are also responsive to proprioceptive inputs (Harrison and Jankowska, 1984; Riddell et al., 1994). Furthermore, there is neuroanatomical evidence that cervical dorsal horn neurons project directly to the pontine $\mathrm{PB} / \mathrm{KF}$ complex in rats (Cechetto et al., 1985; Bernard et al., 1995; Feil and Herbert, 1995), cats (Takeuchi et al., 1995), and monkeys (Craig, 1995). Thus, an anatomical substrate exists for ascending pathway communication to the $\mathrm{PB} / \mathrm{KF}$ nuclei that, according to physiological data from both the newborn (this study) and juvenile rat (Potts et al., 2005), is ultimately responsible for transmitting spinal proprioceptive information to the medullary respiratory centers (Figs. 6-8).

In addition to serving as a target for diverse sensory modalities, including somatic, visceral, and nociceptive afferent information (Jiang et al., 2004), the $\mathrm{PB} / \mathrm{KF}$ is now recognized to be strongly implicated in respiratory control, and especially in the transition between inspiratory and expiratory phases (Dutschmann and Herbert, 2006; Mörschel and Dutschmann, 2009). The reciprocal synaptic connectivity of the $\mathrm{PB} / \mathrm{KF}$ with the ventrolateral medulla (Herbert and Saper, 1990; Ezure, 2004; Ezure and Tanaka, 2006) where the respiratory centers are located (Smith et al., 1991; Mellen et al., 2003; Onimaru and Homma, 2003; Barnes et al., 2007), further implicates these pontine nuclei in the organization of breathing. Therefore, in a manner equivalent to its role in the interactions between respiration and other functions such as nociception (Jiang et al., 2004) and vocalization (Smotherman et al., 2006), the PB/KF complex could additionally serve as an important integrating locus for coordinating mammalian breathing with locomotion.

Our evidence from calcium imaging and selective electrolytic ablations for the essential mediating role played by the $\mathrm{PB} / \mathrm{KF}$ complex corresponds closely to the findings of Potts and colleagues using $\mathrm{PB}$ pharmacological inactivation. A notable difference, however, is that a unilateral blockade of the PB nucleus in the juvenile rat is evidently sufficient to block afferent-evoked respiratory entrainment (Potts et al., 2005), whereas the neonatal $\mathrm{PB} / \mathrm{KF}$ region, which is clearly activated by DR stimulation (Fig. 7), requires bilateral lesioning to eliminate entrainment (Fig. 8). This difference may derive from the different experimental approaches used in the two studies, or it may be attributable to a maturational variability in the respiratory and/or sensory systems of these animals at different stages of development.

Finally, although spinal and pontine synaptic relays are necessary for locomotor-respiratory coupling, their precise contribution to the coordinating process remains unknown. In our in vitro experiments, respiratory rhythm entrainment was achieved by stimulating individual DRs. During actual locomotion, however, the CNS receives a multitude of somatic afferent signals arising from antagonistic flexor and extensor movements of each of the four limbs, and which therefore occur in complex aggregate patterns of synchronous and alternating discharge. It is perhaps in this context that the spinal and pontine relays could be of primary importance by providing sites at which inappropriately timed sensory information is gated out while remaining ascending signals are tuned into effective commands for the cycle-bycycle regulation of respiratory rhythmogenesis.

\section{References}

Barnes BJ, Tuong CM, Mellen NM (2007) Functional imaging reveals respiratory network activity during hypoxic and opioid challenge in the neonate rat tilted sagittal slab preparation. J Neurophysiol 97:2283-2292.

Bernard JF, Dallel R, Raboisson P, Villanueva L, Le Bars D (1995) Organization of the efferent projections from the spinal cervical enlargement to the parabrachial area and periaqueductal gray: a PHA-L study in the rat. J Comp Neurol 353:480-505.

Berry MS, Pentreath VW (1976) Criteria for distinguishing between monosynaptic and polysynaptic transmission. Brain Res 105:1-20.

Boggs DF (2002) Interactions between locomotion and ventilation in tetrapods. Comp Biochem Physiol A Mol Integr Physiol 133:269-288.

Boggs D, Seveyka J, Kilgore D, Dial K (1997) Coordination of respiratory cycles with wingbeat cycles in the black-billed magpie (Pica pica). J Exp Biol 200:1413-1420.

Bramble DM, Carrier DR (1983) Running and breathing in mammals. Science 219:251-256.

Brocard F, Vinay L, Clarac F (1999) Development of hindlimb postural control during the first postnatal week in the rat. Brain Res Dev Brain Res 117:81-89.

Cechetto DF, Standaert DG, Saper CB (1985) Spinal and trigeminal dorsal horn projections to the parabrachial nucleus in the rat. J Comp Neurol 240:153-160.

Chen C, Zhou XF, Rush RA (1996) Neurotrophin-3 and trkCimmunoreactive neurons in rat dorsal root ganglia correlate by distribution and morphology. Neurochem Res 21:809-814.

Clarac F, Cattaert D, Le Ray D (2000) Central control components of a 'simple' stretch reflex. Trends Neurosci 23:199-208.

Craig AD (1995) Distribution of brainstem projections from spinal lamina I neurons in the cat and the monkey. J Comp Neurol 361:225-248.

Craig AD Jr, Tapper DN (1978) Lateral cervical nucleus in the cat: functional organization and characteristics. J Neurophysiol 41:1511-1534.

Decherchi P, Dousset E, Jammes Y (2007) Respiratory and cardiovascular responses evoked by tibialis anterior muscle afferent fibers in rats. Exp Brain Res 183:299-312.

Djouhri L, Brown AG, Short AD (1994) Differential ascending projections from neurons in the cat's lateral cervical nucleus. Exp Brain Res 101:375-384.

Dutschmann M, Herbert H (2006) The Kolliker-Fuse nucleus gates the postinspiratory phase of the respiratory cycle to control inspiratory offswitch and upper airway resistance in rat. Eur J Neurosci 24:1071-1084.

Duysens J, Clarac F, Cruse H (2000) Load-regulating mechanisms in gait and posture: comparative aspects. Physiol Rev 80:83-133.

Ezure K (2004) Respiration-related afferents to parabrachial pontine regions. Respir Physiol Neurobiol 143:167-175.

Ezure K, Tanaka I (2006) Distribution and medullary projection of respiratory neurons in the dorsolateral pons of the rat. Neuroscience 141:1011-1023.

Falgairolle M, Cazalets JR (2007) Metachronal coupling between spinal neuronal networks during locomotor activity in newborn rat. J Physiol 580:87-102.

Falgairolle M, de Seze M, Juvin L, Morin D, Cazalets JR (2006) Coordinated network functioning in the spinal cord: an evolutionary perspective. J Physiol Paris 100:304-316.

Feil K, Herbert H (1995) Topographic organization of spinal and trigeminal somatosensory pathways to the rat parabrachial and Kolliker-Fuse nuclei. J Comp Neurol 353:506-528.

Funk GD, Milsom WK, Steeves JD (1992) Coordination of wingbeat and respiration in the Canada goose. I. Passive wing flapping. J Appl Physiol 73:1014-1024. 
Gariépy JF, Missaghi K, Chevallier S, Chartré S, Robert M, Auclair F, Lund JP, Dubuc R (2012) Specific neural substrate linking respiration to locomotion. Proc Natl Acad Sci U S A 109:E84-E92.

Giraudin A, Cabirol-Pol MJ, Simmers J, Morin D (2008) Intercostal and abdominal respiratory motoneurons in the neonatal rat spinal cord: spatiotemporal organization and responses to limb afferent stimulation. J Neurophysiol 99:2626-2640.

Grélot L, Milano S, Portillo F, Miller AD, Bianchi AL (1992) Membrane potential changes of phrenic motoneurons during fictive vomiting, coughing, and swallowing in the decerebrate cat. J Neurophysiol 68:2110-2119.

Harrison PJ, Jankowska E (1984) An intracellular study of descending and non-cutaneous afferent input to spinocervical tract neurones in the cat. J Physiol 356:245-261.

Herbert H, Saper CB (1990) Cholecystokinin-, galanin-, and corticotropinreleasing factor-like immunoreactive projections from the nucleus of the solitary tract to the parabrachial nucleus in the rat. J Comp Neurol 293:581-598.

Hilaire G, Morin D, Lajard AM, Monteau R (1993) Changes in serotonin metabolism may elicit obstructive apnoea in the newborn rat. J Physiol 466:367-381.

Howard P, Bromberger-Barnea B, Fitzgerald RS, Bane HN (1969) Ventilatory responses to peripheral nerve stimulation at different times in the respiratory cycle. Respir Physiol 7:389-398.

Ikegaya Y, Le Bon-Jego M, Yuste R (2005) Large-scale imaging of cortical network activity with calcium indicators. Neurosci Res 52:132-138.

Iscoe S, Polosa C (1976) Synchronization of respiratory frequency by somatic afferent stimulation. J Appl Physiol 40:138-148.

Jamon M, Clarac F (1998) Early walking in the neonatal rat: a kinematic study. Behav Neurosci 112:1218-1228.

Jiang M, Alheid GF, Calandriello T, McCrimmon DR (2004) Parabrachiallateral pontine neurons link nociception and breathing. Respir Physiol Neurobiol 143:215-233.

Juvin L, Morin D (2005) Descending respiratory polysynaptic inputs to cervical and thoracic motoneurons diminish during early postnatal maturation in rat spinal cord. Eur J Neurosci 21:808-813.

Juvin L, Simmers J, Morin D (2005) Propriospinal circuitry underlying interlimb coordination in mammalian quadrupedal locomotion. J Neurosci 25:6025-6035.

Juvin L, Simmers J, Morin D (2007) Locomotor rhythmogenesis in the isolated rat spinal cord: a phase-coupled set of symmetrical flexion extension oscillators. J Physiol 583:115-128.

Juvin L, Le Gal JP, Simmers J, Morin D (2012) Cervico-lumbar coordination in mammalian quadrupedal locomotion: role of spinal thoracic circuitry and limb sensory inputs. J Neurosci 32:953-965.

Kajander KC, Giesler GJ Jr (1987) Responses of neurons in the lateral cervical nucleus of the cat to noxious cutaneous stimulation. J Neurophysiol 57:1686-1704.

Kudo N, Yamada T (1987) Morphological and physiological studies of development of the monosynaptic reflex pathway in the rat lumbar spinal cord. J Physiol 389:441-459.

Luppi PH, Aston-Jones G, Akaoka H, Chouvet G, Jouvet M (1995) Afferent projections to the rat locus coeruleus demonstrated by retrograde and anterograde tracing with cholera-toxin B subunit and Phaseolus vulgaris leucoagglutinin. Neuroscience 65:119-160.

Manogue KR, Paton JA (1982) Respiratory gating of activity in the avian vocal control system. Brain Res 247:383-387.

McFarland DH, Lund JP (1995) Modification of mastication and respira- tion during swallowing in the adult human. J Neurophysiol 74:1509-1517.

Mellen NM, Janczewski WA, Bocchiaro CM, Feldman JL (2003) Opioidinduced quantal slowing reveals dual networks for respiratory rhythm generation. Neuron 37:821-826.

Metherate RS, da Costa DC, Herron P, Dykes RW (1986) A thalamic terminus of the lateral cervical nucleus: the lateral division of the posterior nuclear group. J Neurophysiol 56:1498-1520.

Morin D, Viala D (2002) Coordinations of locomotor and respiratory rhythms in vitro are critically dependent on hindlimb sensory inputs. J Neurosci 22:4756-4765.

Morin D, Bonnot A, Ballion B, Viala D (2000) alpha1-adrenergic receptorinduced slow rhythmicity in nonrespiratory cervical motoneurons of neonatal rat spinal cord. Eur J Neurosci 12:2950-2966.

Mörschel M, Dutschmann M (2009) Pontine respiratory activity involved in inspiratory/expiratory phase transition. Philos Trans R Soc Lond B Biol Sci 364:2517-2526.

Onimaru H, Homma I (2003) A novel functional neuron group for respiratory rhythm generation in the ventral medulla. J Neurosci 23:1478-1486.

Orlovsky GN, Deliagina TG, Grillner S (1999) Neuronal control of locomotion: from mollusc to man. New York: Oxford UP.

Palisses R, Persegol L, Viala D, Viala G (1988) Reflex modulation of phrenic activity through hindlimb passive motion in decorticate and spinal rabbit preparation. Neuroscience 24:719-728.

Pearson K (2000) Motor systems. Curr Opin Neurobiol 10:649-654.

Persegol L, Palisses R, Viala D (1993) Characterization of hindlimb muscle afferents involved in ventilatory effects observed in decerebrate and spinal preparations. Exp Brain Res 92:495-501.

Potts JT, Rybak IA, Paton JF (2005) Respiratory rhythm entrainment by somatic afferent stimulation. J Neurosci 25:1965-1978.

Riddell JS, Jankowska E, Hammar I, Szabo-Läckberg Z (1994) Ascending tract neurones processing information from group II muscle afferents in sacral segments of the feline spinal cord. J Physiol 475:469-481.

Smith JC, Ellenberger HH, Ballanyi K, Richter DW, Feldman JL (1991) PreBotzinger complex: a brainstem region that may generate respiratory rhythm in mammals. Science 254:726-729.

Smotherman M, Kobayasi K, Ma J, Zhang S, Metzner W (2006) A mechanism for vocal-respiratory coupling in the mammalian parabrachial nucleus. J Neurosci 26:4860-4869.

Takeuchi Y, Itoh M, Miki T, Chen XH, Sun W (1995) Hypoglossal afferents to lamina I neurons of the cervical spinal cord projecting to the parabrachial nucleus in the cat. Somatosens Mot Res 12:191-198.

Vinay L, Cazalets JR, Clarac F (1995) Evidence for the existence of a functional polysynaptic pathway from trigeminal afferents to lumbar motoneurons in the neonatal rat. Eur J Neurosci 7:143-151.

Waisbren SJ, Whiting CS, Nadel ER (1990) Effects of passive limb movement on pulmonary ventilation. Yale J Biol Med 63:549-556.

Wang T, Carrier DR, Hicks JW (1997) Ventilation and gas exchange in lizards during treadmill exercise. J Exp Biol 200:2629-2639.

Westerga J, Gramsbergen A (1990) The development of locomotion in the rat. Brain Res Dev Brain Res 57:163-174

Yuste R, Katz LC (1991) Control of postsynaptic $\mathrm{Ca}^{2+}$ influx in developing neocortex by excitatory and inhibitory neurotransmitters. Neuron 6:333-344.

Zhuang J, Xu F, Zhang C, Frazier DT (2009) Passive limb movement augments ventilatory response to $\mathrm{CO}_{2}$ via sciatic inputs in anesthetized rats. Respir Physiol Neurobiol 167:174-180. 